\title{
Retroperitoneal Lymph Node Dissection with Concomitant IVC Thrombectomy, Caval Wall Resection, and Grafting for Metastatic NSGCT
}

Tariq Hakky, Timothy Kim, Alejandro R. Rodriguez, Paul Armstrong, Devanand Mangar, Philippe E. Spiess

Department of Urology (TH, TK, ARR) and Department of Vascular and Endovascular Surgery (PA) University of South Florida, Department of Anesthesia (DM), Tampa General Hospital and Department of Genitourinary Oncology (PES), Moffitt Cancer Center, Tampa, FL, USA

\begin{abstract}
Introduction: The management of a post-chemotherapy retroperitoneal mass secondary to testicular cancer can present a surgical challenge when involving adjacent organs or major vascular structures. We present the first video of a retroperitoneal lymph node dissection (RPLND) with IVC (inferior vena cava) thrombectomy, caval wall resection resulting from metastatic non-seminomatous germ cell testis (NSGCT) cancer.

Methods: In this surgical video, we highlight important surgical considerations in the management of a postchemotherapy retroperitoneal mass with direct IVC wall invasion and level 2 thrombus in such a patient.

Results: A 34 year old man underwent a right inguinal orchiectomy for a mixed NSGCT (embryonal, yolk sac, and teratoma components) and elevated serum tumor markers. He underwent systemic chemotherapy (BEP regimen $\mathrm{x} 4$ cycles) with subsequent near normalization of tumor markers. His post-chemotherapy imaging revealed a $6 \mathrm{~cm}$ residual retroperitoneal mass with a level 2 IVC tumor thrombus and suspected direct infrarenal IVC wall invasion from the mass. The patient underwent an open post-chemotherapy RPLND, IVC thrombectomy, IVC resection and grafting. The final pathology report of the retroperitoneal mass revealed teratoma with no viable germ cell tumor elements and negative surgical margins. His intra-operative and post-operative stages were unremarkable with his IVC graft remaining patent and no evidence of disease recurrence at last follow-up. Conclusion: We present the first surgical video of a post-chemotherapy RPLND with IVC thrombectomy, caval wall resection and grafting for metastatic NSGCT. The final pathology report of teratoma with no viable tumor highlights the local vascular invasive potential of such pathology.
\end{abstract}

\section{ARTICLE INFO}

Int Braz J Urol. 2012; 38 (Video \#1): 135_136

Available at: www.brazjurol.com.br/videos/january_february_2012/Hakky_135_136video.htm

Accepted:

January 20, 2012

Correspondence address:

Dr. Philippe E. Spiess

Department of Urology

H Lee Moffitt Cancer Center

E-mail: Philippe.Spiess@moffitt.org 


\section{EDITORIAL COMIMENT}

Full bilateral RPLND is the standard of care for patients with residual retroperitoneal masses post chemotherapy. With proper and meticulous surgical technique, the morbidity of the procedure is minimal and the in-field recurrences are negligible. In the following video, Hakki et al. elegantly demonstrates that resection of ALL residual masses within the retroperitoneum is important for long-term disease control even if it requires major vessel resection. IVC thrombus in the setting of metastatic testes cancer is rare and can develop either through retrograde spread of disease via the gonadal vein or direct invasion of the IVC wall by the retroperitoneal mass. The decision of IVC grafting is challenging as one has to balance the benefit of maintaining a patent IVC versus the high risk of clotting off the graft and lifelong anticoagulation in such a young patient. Take into account the above mentioned, the other reasonable option is to consider ligation of the IVC completely, particularly when a patient already have developed collaterals due to obstruction of the IVC with the tumor thrombus.
Dr. Wassim Kassouf Assistant Professor of Urology McGill University Montreal, Canada E-mail:wassim.kassouf@muhc.mcgill.ca 